\title{
Organochlorine Pesticides and PCBs in Traditionally and Industrially Smoked Pork Meat Products from Bosnia and Herzegovina
}

\author{
Brankica Kartalović ${ }^{1}$, Krešimir Mastanjević ${ }^{2, *}$,, Nikolina Novakov ${ }^{3}$, Jelena Vranešević ${ }^{1}$, \\ Dragana Ljubojević Pelić ${ }^{1}$ (D), Leona Puljić ${ }^{4}$ and Kristina Habschied ${ }^{2}$ (I) \\ 1 Scientific Veterinary Institute Novi Sad, Rumenački put 20, 21000 Novi Sad, Serbia; \\ brankica.kartalovic@gmail.com (B.K.); vet.bmv@hotmail.com (J.V.); \\ ljubojevic.ljubojevicd.dragana@gmail.com (D.L.P.) \\ 2 Faculty of Food technology, Josip Juraj Strossmayer University of Osijek, F. Kuhača 20, 31000 Osijek, Croatia; \\ kristinahabschied@gmail.com \\ 3 Faculty of Agriculture Novi Sad, Trg Dositeja Obradovića 8, 21000 Novi Sad, Serbia; nikolina@polj.uns.ac.rs \\ 4 The Faculty of Agriculture and Food Technology (APTF) of the University of Mostar, Biskupa Čule bb, \\ 88000 Mostar, Bosnia and Herzegovina; leonapuljic224@gmail.com \\ * Correspondence: kmastanj@gmail.com; Tel.: +385-3122-4300
}

Received: 24 December 2019; Accepted: 15 January 2020; Published: 17 January 2020

\begin{abstract}
The aim of this study was to determine the concentration of 19 organochlorine pesticides (OCPs): (hexachlorocyclohexane $(\alpha-\mathrm{HCH}, \beta-\mathrm{HCH}, \delta-\mathrm{HCH})$, lindane, aldrin, heptachlor, heptachlor epoxide, trans-chlordane, cis-chlordane, endosulfane I, endosulfane II, endosulfane sulfate, dichlorodiphenyltrichloroethane (DDT), dichlorodiphenyldichloroethylene (DDE), dieldrin, endrin, dichlorodiphenyldichloroethane (DDD), methoxychlor and endrin ketone and 6 polychlorinated biphenyls (PCBs) (PCB 28, PCB 52, PCB 101, PCB 153, PCB 138 and PCB 180). The samples were taken from pancetta, dry pork neck (budiola), pork tenderloin and sausages produced in Rakitno (Bosnia and Herzegovina), smoked in both a traditional smokehouse and in an industrial chamber. Instrumental analysis was performed using gas chromatography-mass spectrometry (GC-MS). The reliability of the results, i.e., quality control is ensured by standard laboratory practice, which involves participation in proficiency test, the use of blank samples, reference materials and implementation of recommendations given by the relevant international organizations. The concentrations of $\alpha-\mathrm{HCH}$, lindane, PCB 28, PCB 52 and PCB 153 were detected and quantified. The concentrations of OCPs and PCBs did not significantly vary depending on product type and the conditions of production. All the examined samples were for human consumption.
\end{abstract}

Keywords: OCPs; PCBs; smoked pork meat products; pancetta; pork neck; pork tenderloin; sausage; traditional and industrial smoking

\section{Introduction}

In line with the current trend aimed at revitalizing and supporting traditional food manufacturing processes, autochthonous meat products made from locally available raw materials are becoming more and more significant. Smoking is a traditional method of food preservation and is a quite important in many countries worldwide. Smoked meat products in Bosnia and Herzegovina and the Balkans region alike are made in small smokehouses using open fire. Smoking is used not only to contribute the preservation and extension of shelf life of products, but also to affect sensorial properties of final products [1-4]. It should be pointed out, however, that the consumption of food produced in a traditional manner carries certain food safety risks. Consumption of foods containing chemical 
contaminants such as organochlorine pesticides (OCPs) and polychlorinated biphenyls (PCBs) can lead to intoxication, mainly due to long-term exposure [5]. In case of smoked products, the greatest attention is paid to the content of polycyclic aromatic hydrocarbons (PAHs), which are discussed in numerous publications [1,2,6-9], while little attention is paid to the content of OCPs and PCBs, whose effects on human health are certainly not negligible. PCBs, DDT, $\mathrm{HCH}$ gamma isomer (lindane) are potentially carcinogenic, according to the International Agency for Research on Cancer (IARS). IARS listed PCBs in group I, which is made up of the most carcinogenic compound for humans [10]. In addition, very low concentrations of PCBs are known to cause adverse immunotoxicity and neurotoxicity effects in humans [11]. OCPs and PCBs were used in agricultural and industrial activities. OCPs have been extensively used for pest management in agriculture mainly due to their low cost but also high efficiency [5]. PCBs have been widely used as additives in industrial materials including plastics, paints and paper. Moreover, PCBs have been used in electronic industry like transformers and capacitors due to their low electrical conductivity and high thermo-resistance [10]. Because of their adverse effects on human health and environment the usage of most OCPs and PCBs were banned in many countries. However, these compounds are still widely detected both in environment and consequently in food.

It is known that the above-mentioned contaminants are very persistent, characterized by high lipophilic properties, and therefore accumulate in food chain, especially in fats. Consequently, food of animal origin is their main source $[12,13]$. Many people are most exposed to these compounds $(>90 \%)$ through the food chain, while inhalation or skin exposure is significantly lower $[14,15]$. The control of OCPs and PCBs residue in food is important for consumer safety. Different organizations, such as regulatory, advisory and scientific bodies specified different maximum residue levels (MRLs) as well as acceptable maximum intake for these compounds. Numerous analytical methods for the analysis of OCPs and PCBs are widely available. Muir and Sverko [16] highlighted the importance of modern capillary gas chromatography (GC) equipment with either electron capture or low-resolution mass spectrometry (MS) detection for separation and quantification of OCPs and PCBs. There are also different screening methods such as commercially available enzyme-linked immuno-absorbent assays. Different methods for extracting OCPs and PCBs such as liquid:liquid (LLE), cavity-dispersed microwave-assisted (MAE), focused microwave-assisted (FME), solid-phase (SPE), and pressurized fluid (PFE) extraction techniques were previously described [17]. Limits of detection (LODs) and limits of quantification (LOQs) may vary between different methods and between different laboratories.

The aim of this study was the examination of chemical safety of pork meat products smoked in traditional and in industrial conditions. The parameters used to assess chemical safety were OCPs and PCBs. The analyzed PCBs (PCBs 28, 52, 101, 138, 153 and 180) belong to the so-called indicator PCBs that have been proposed to be monitored by several countries and international organizations [18]. The reliability and accuracy of analytical results were achieved by using a GC-MS method that was developed and validated as previously described [19].

\section{Materials and Methods}

\subsection{Preparation of Samples and Smoking Procedures}

All samples of pancetta, dry pork neck (budiola), pork tenderloin and sausages were produced in a local meat industry, which is located in the Municipality of Posušje, Rakitno, Bosnia and Herzegovina. The raw material used for the production of these meat products originated from local meat industry farms. Raw materials were processed using traditional technology. A part of a pig carcass was cut for the production of pancetta in such way that a part of the chest surrounded by the ribs with a big belly was separated from the back by a longitudinal incision and from the neck with cross-section between the third and fourth rib. The bones and cartilage ribs were left as a part of chest. Corresponding part of the belly was separated from the rest of the carcass by transverse section at the level of the lumbal part of spine. Rib bones and cartilage were separated from the chest muscles. The samples of 
pancetta had a rectangular shape. The samples of budiola were made from previously formed pieces of pork necks. The samples of tenderloin were produced from long dorsal muscle musculus longissimus dorsi. After determining the weight of each individual sample, they were salted using a mixture of a mineral and a nitrite salt in a 50:50 ratio. The salting was done manually by using unspecified amount of salt put on the surface of meat in a cooling chamber at the temperature of $+4{ }^{\circ} \mathrm{C}$ in duration of seven days. The samples were then rinsed with water and moved to a place for drying and smoking where they were dried and tempered for 12 to $20 \mathrm{~h}$. The samples were smoked in both a traditional smokehouse (open combustion chamber) and in an industrial chamber produced by Maurer-Atmos Middleby GmbH., (Reichenau, Germany). In traditional smokehouse, the smoking process for all products lasted 20 days. The traditional products were smoked for $6-8 \mathrm{~h}$ per day for first 6 days and after that for 2-3 h every second or third day for 14 days. The industrial products were smoked for $4 \mathrm{~h}$ per day for 3 days in the industrial chamber. Experimental conditions during smoking procedures for both traditional and industrial conditions are shown in Table 1. The total time of sample preparation was for 45 days for both methods. Traditional sausages were made using the local recipe, from the mixture of the first-category meat with the addition of $30 \%$ of the second-category meat, mineral salt, sweet and hot peppers and minced garlic. The mixture was stuffed in natural casings. Smoking was performed in both a traditional smokehouse and in an industrial chamber. In traditional smokehouse, smoking lasted 20 days and in the industrial chamber for 3 days. The total length of production for industrial and traditional production was 30 days.

Table 1. Experimental conditions during smoking procedures of pork meat products.

\begin{tabular}{ccccc}
\hline Sample & Number of Samples & Production Conditions & Smoking Duration (days) & Duration of Production \\
\hline Pancetta & 12 & Traditional & $20^{1}$ & 45 \\
Pancetta & 12 & Industrial & $3{ }^{2}$ & 45 \\
Tenderloin & 12 & Traditional & 20 & 45 \\
Tenderloin & 12 & Industrial & 3 & 45 \\
Budiola & 12 & Traditional & 20 & 45 \\
Budiola & 12 & Industrial & 3 & 30 \\
Sausage & 12 & Traditional & 20 & 30 \\
Sausage & 12 & Industrial & 3 & 35 \\
\hline
\end{tabular}

${ }^{1}$ first 6 days for $6-8 \mathrm{~h}$ per day, 14 days every two or three days for $2-3 \mathrm{~h} ;{ }^{2} 4 \mathrm{~h}$ per day.

\subsection{Reagents and Materials}

All chemicals and reagents used were of analytical grade with high purity. Calibration solutions were prepared using the pesticides mix of 20 pesticides-Organochlorine pesticides mixture produced by Ultra Scientific Inc., (Nort Kingstown, RI, USA), lot CL-1069; PCB Mix 1 that include PCB 28, PCB 52, PCB 101, PCB 153, PCB 138 and PCB 180, produced by Dr Ehrenstorfer, lot G126821IO (Bgm.Schlosser-StraBe 6A-Ausburg, Germany). In order to eliminate the influence of the matrix, calibration through matrix blank sample was performed according to European Commission Document No. SANCO /12571/2013 [20].

Working standard solution of Pesticides mix that contains 19 pesticides-Chlorinated Pesticides-herbicides lot: 213091108, was obtained from AccuStandard Inc. (New Haven, CT, USA), used for spike preparation for internal control. Spiked samples were used with the purpose of internal control of the following parameters: recovery, precision, limit of quantification (LOQ) and limit of detection (LOD). Chromatogram of the standard mixtures is shown in Figure 1. 


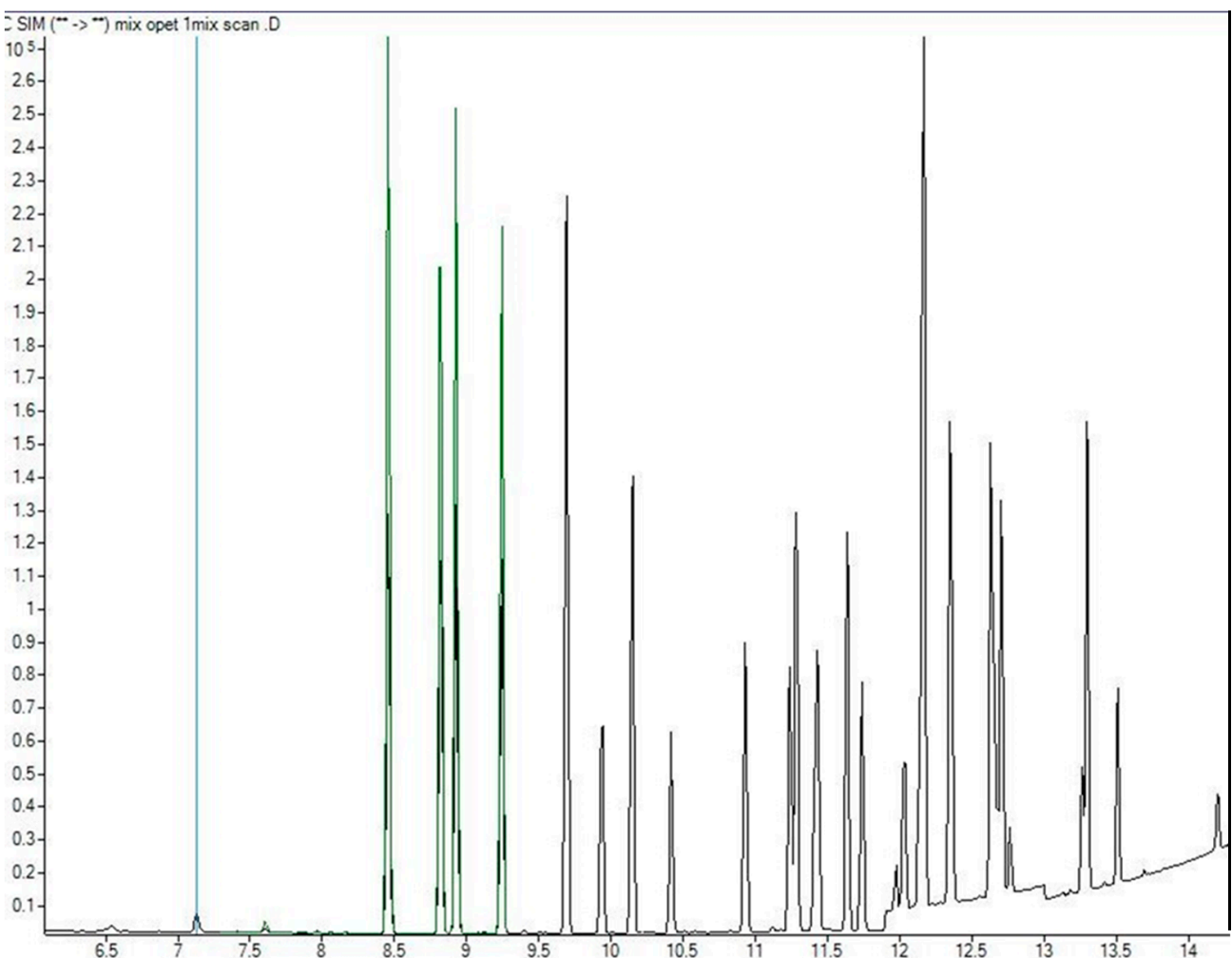

Figure 1. Chromatogram of the standard mixture.

\subsection{Sample Preparation}

The method of sample preparation was based on the extraction with acetonitrile (ACN) produced by Sigma-Aldrich (St. Louis, MI, USA) in the presence of anhydrous magnesium sulfate $\left(\mathrm{MgSO}_{4}\right)$ and anhydrous sodium acetate $\left(\mathrm{CH}_{3} \mathrm{COONa}\right)$, produced by Merck (Darmstadt, Germany). Sample (3 g) was measured and transferred into centrifuge tube and $3 \mathrm{~mL}$ of water and $3 \mathrm{~mL}$ of acetonitrile were added. After intensive stirring on a vortex, $3 \mathrm{~g}$ of anhydrous magnesium sulfate and $1 \mathrm{~g}$ of anhydrous sodium acetate were added. Exothermic reaction occurred within $1 \mathrm{~min}$ after the intense stirring on vortex. The sample was then centrifuged for $5 \mathrm{~min}$ at $1110 \times g$ (approximately $3000 \mathrm{rpm}$ ). $1 \mathrm{~mL}$ of upper acetonitrile extract was transferred into a $5 \mathrm{~mL}$ tube, which contained $150 \mathrm{mg}$ of anhydrous magnesium sulfate, $100 \mathrm{mg}$ of primary and secondary amine (PSA) and $50 \mathrm{mg}$ of C18 [21-23]. The tube content was centrifuged for $5 \mathrm{~min}$ at $1110 \times \mathrm{g}$. After centrifuging, purified and clear extract was obtained. After that, $0.5 \mathrm{~mL}$ of the extract was evaporated in nitrogen and reconstituted with hexane. The sample prepared in this way was ready for the analysis on GCMS-Agilent 7890B/5977A obtained from Agilent Technologies Inc., (Santa Clara, CA, USA).

\subsection{GCMS Analysis and Instrumentation}

The identification of OC pesticides was based on a comparison of retention times of the peaks and target ions with those obtained from a standard mixture of OC pesticides (standards supplied by instrument manufacturer). The quantification was based on external calibration curves prepared from the standard solution of each of the pesticides.

The gas-mass chromatography was Agilent 7890B/5977A MSD (Santa Clara, CA, USA). The GC operating conditions are shown in Table 2. The verification of the peaks was carried out, based on the retention times and target ions compared to those of external OC pesticides. Procedural blank and solvent blanks were analyzed and quantified, but no OC pesticides were found in these blanks. Determination was made in splitless mode, because of that we don't have split mode, carrier gas was Helium, velocity-32.098 cm/sec; pressure-7.0 psi. Determination was made at constant flow. 
Table 2. The GC operating conditions.

\begin{tabular}{cl}
\hline Descriptions & \multicolumn{1}{c}{ Conditions } \\
\hline Instrument & Agilent 7890B/5977A MSD (Santa Clara, CA, USA) \\
\hline Column & $\begin{array}{l}\text { Fused silica column }(30 \mathrm{~m} \times 0.25 \mu \mathrm{m} \text { film of HP-5M-thickness) Agilent Technologies, Inc., } \\
\text { (Santa Clara, CA, USA) }\end{array}$ \\
\hline \multirow{2}{*}{ Temperature } & Injection $280{ }^{\circ} \mathrm{C}$ \\
\cline { 2 - 2 } & MSD $280^{\circ} \mathrm{C}$ \\
\cline { 2 - 2 } & $\begin{array}{l}\text { Column } 50{ }^{\circ} \mathrm{C}\left(0.4 \mathrm{~min} \text { hold) to } 195^{\circ} \mathrm{C} \text { at } 25^{\circ} \mathrm{C} / \mathrm{min} ;\right. \\
\text { hold to } 265{ }^{\circ} \mathrm{C} \text { for } 1.5 \mathrm{~min} \text { at } 8{ }^{\circ} \mathrm{C} / \mathrm{min} \text {; maintained at } 315^{\circ} \mathrm{C} \text { for } 1.25 \mathrm{~min} \text { at } 20^{\circ} \mathrm{C} / \mathrm{min}\end{array}$ \\
\hline Carrier gas & Helium \\
\hline Injection volume & $4 \mu \mathrm{L}$ \\
\hline
\end{tabular}

\subsection{Accuracy and Precision}

The accuracy of the method was calculated as percent recovery of pesticides from spiked samples. $3 \mathrm{~g}$ of homogenized sample was spiked prior to the determination procedure by adding a mixed pesticide standard working solution to reach the final fortification levels of 5, 10, 50, 100 and $500 \mu \mathrm{g} \mathrm{kg}^{-1}$. For each level, five replicates were analyzed. After the addition of each concentration in the matrix, the mixture was equilibrated by shaking, and the samples were allowed to settle for $30 \mathrm{~min}$ prior to extraction in order to ensure the sufficient contact of the analytes with the whole matrix. Then, the samples were prepared according to the method which was described earlier.

The precision in case of repeatability (RSDr) was determined at fortification levels of $0.05 \mathrm{mg} \mathrm{kg}^{-1}$ with five replicates on the same day. Precision in case of reproducibility (RSDR) was determined at fortification levels of $0.05 \mathrm{mg} \mathrm{kg}^{-1}$ with five replicates at three-week intervals. The limit of detection (LOD) was calculated according to Magnusson and Örnemark [24]. In order to determine the LOD of each analyte, ten independent sample blanks fortified at the lowest acceptable concentration of $0.005 \mathrm{mg} \mathrm{kg}^{-1}$ were injected, and the LOD was expressed as the analyte concentration corresponding to three times the standard deviation. Limit of quantification (LOQ) was determined according to European Commission Document SANCO/12571/2013 [20]. LOQ was set as the lowest fortification level for each pesticide that was achieved in the acceptable accuracy (mean recoveries for individual pesticides in the range of $70-130 \%$ ) and precision ( $R S D r \leq 20 \%$ ).

\subsection{Statistical Analysis}

Data analysis was performed using Statistica version 12 from StatSoft ${ }^{\circledR}$ (Tulsa, OK, USA) and Excel (Microsoft Excel, 2007) to determine the descriptive statistic parameters (mean, standard deviation, range) and one-way analysis of variance (ANOVA). ANOVA was used for the assessment of variation in different meat products before smoking and after smoking. Post-hoc Tukey's test was used for statistical analysis of differences with a statistical significance defined at $p<0.05$.

\section{Results}

\subsection{Method Validation}

Method validation and quality control were conducted following the European Commission SANTE /11813/2017 [25]. The method was validated in terms of the optimal linearity $\left(r^{2}>0.99\right)$. Precision was evaluated by repeatability in triplicate $\left(50.0 \mu \mathrm{g} \mathrm{kg}^{-1}, n=20\right)$ and it ranged from $0.78-17.91 \%$. Recovery ranged from $81.61 \%$ to $116.33 \%$. The limits of quantification were lower than the maximum residual limits prescribed for examined products. The obtained results are shown in Tables 3 and 4. 
Table 3. The average values of LOD, LOQ, precision, linearity, recovery and RSD in blank smoked meat samples, spiked with $50 \mu \mathrm{g} / \mathrm{kg}(n=20)$.

\begin{tabular}{ccccccc}
\hline OCPs & $\begin{array}{c}\text { LOD } \\
(\boldsymbol{\mu g} / \mathbf{k g})\end{array}$ & $\begin{array}{c}\text { LOQ } \\
(\boldsymbol{\mu g} / \mathbf{k g})\end{array}$ & $\begin{array}{c}\text { Precision } \\
\mathbf{( \% )}\end{array}$ & $\begin{array}{c}\text { Linearity } \\
\left(\boldsymbol{r}^{\mathbf{2}} \mathbf{)}\right.\end{array}$ & $\begin{array}{c}\text { Recovery } \\
\mathbf{( \% )}\end{array}$ & $\begin{array}{c}\text { RSD } \\
(\mathbf{\%})\end{array}$ \\
\hline$\alpha-\mathrm{HCH}$ & 1.38 & 4.66 & 4.26 & 0.9991 & 96.13 & 5.26 \\
$\beta-\mathrm{HCH}$ & 0.56 & 1.89 & 17.9 & 0.9992 & 99.12 & 8.89 \\
$\delta$-HCH & 0.28 & 1.18 & 0.78 & 0.9991 & 100.3 & 18.2 \\
Lindane & 0.28 & 1.10 & 8.88 & 0.9993 & 99.50 & 8.28 \\
Heptachlor & 0.27 & 1.10 & 3.36 & 0.9991 & 88.10 & 14.6 \\
Aldrin & 1.44 & 4.64 & 3.62 & 0.9993 & 98.31 & 3.41 \\
Heptachlor epoxide & 0.55 & 1.59 & 3.49 & 0.9991 & 94.39 & 3.38 \\
Trans-chlordane & 0.42 & 1.22 & 4.42 & 0.9991 & 90.22 & 8.23 \\
Cis-chlordane & 1.18 & 3.89 & 4.28 & 0.9990 & 91.56 & 8.18 \\
Endosulfane I & 0.88 & 2.78 & 9.26 & 0.9991 & 87.27 & 8.68 \\
DDE & 1.38 & 4.76 & 3.29 & 0.9990 & 96.87 & 3.88 \\
Dieldrin & 1.49 & 5.20 & 3.48 & 0.9992 & 94.36 & 3.37 \\
Endrin & 0.86 & 3.12 & 8.49 & 0.9998 & 83.36 & 16.2 \\
DDD & 1.22 & 3.89 & 5.72 & 0.9997 & 81.61 & 14.3 \\
Endosulfane II & 1.51 & 4.92 & 7.81 & 0.9991 & 91.21 & 10.3 \\
DDT & 1.38 & 4.78 & 3.48 & 0.9991 & 94.36 & 3.37 \\
Endosulfane sulphate & 1.45 & 4.64 & 13.7 & 0.9994 & 116.3 & 15.3 \\
Metoxichlor & 0.59 & 2.07 & 7.66 & 0.9994 & 106.1 & 1.78 \\
Endrin ketone & 1.26 & 4.36 & 5.59 & 0.9991 & 85.58 & 10.5 \\
\hline
\end{tabular}

LOD-Limit of detection; LOQ-Limit of quantification; $r$-Correlation coefficient; RSD—Precision in case of repeatability; DDE-dichlorodiphenyldichloroethylene; DDD—dichlorodiphenyldichloroethane; DDT—dichlorodiphenyltrichloroethane.

Table 4. The average values of LOD, LOQ, precision, linearity, recovery and RSD in blank smoked meat samples, spiked with $50 \mu \mathrm{g} / \mathrm{kg}(n=20)$.

\begin{tabular}{ccccccc}
\hline PCBs & $\begin{array}{c}\text { LOD } \\
(\mu \mathrm{g} / \mathbf{k g})\end{array}$ & $\begin{array}{c}\text { LOQ } \\
(\mu \mathrm{g} / \mathbf{k g})\end{array}$ & $\begin{array}{c}\text { Precision } \\
(\mathbf{\%})\end{array}$ & $\begin{array}{c}\text { Linearity } \\
\left(\boldsymbol{r}^{\mathbf{2}}\right)\end{array}$ & $\begin{array}{c}\text { Recovery } \\
(\mathbf{\%})\end{array}$ & $\begin{array}{c}\text { RSD } \\
(\mathbf{\%})\end{array}$ \\
\hline PCB 28 & 0.6 & 1.9 & 3.4 & 0.9994 & 106.3 & 3.7 \\
PCB 52 & 1.0 & 4.6 & 7.8 & 0.9992 & 101.4 & 8.6 \\
PCB 101 & 1.0 & 3.7 & 6.4 & 0.9991 & 101.1 & 7.7 \\
PCB 138 & 1.0 & 3.2 & 6.4 & 0.9990 & 99.80 & 7.7 \\
PCB 153 & 0.9 & 3.1 & 5.5 & 0.9991 & 110.9 & 8.3 \\
PCB 180 & 1.2 & 4.0 & 7.3 & 0.9981 & 112.3 & 10 \\
\hline
\end{tabular}

LOD—Limit of detection; LOQ—Limit of quantification; $r$-Correlation coefficient; RSD—Precision in case of repeatability.

In calculating measurement uncertainty, the contributions PT (FAPAS: Pesticides and PCB in Milk Powder, July-September 2019, Round 05136), the contribution of reproducibility and contribution of bias were taken into account.

\subsection{Concentrations of OCPs and PCBs}

The study results are presented in Table 5. The concentrations of OCPs and PCBs for each product were examined before smoking, after traditional and industrial smoking and at the end of the manufacturing process. For each product the concentrations of $\beta-\mathrm{HCH}, \delta-\mathrm{HCH}$, heptachlor, aldrin, heptachlor epoxide, trans-chlordane, cis-hlordane, endosulfane I, DDE, dieldrin, endrin, DDD, endosulfan II, DDT, endosulfan sulphate, metoxichlor, endrin keton as well as PCB 101, PCB 138 and PCB 180 were under the limit of detection and limit of quantification in all examined samples. Concentrations of $\alpha-\mathrm{HCH}$, lindane, PCB 28, PCB 52 and PCB 153 were detected and quantified. All these values are below the prescribed maximum residual levels according to European Union legislation [26-29]. Statistical analysis revealed that there are no statistically significance differences between examined pork meat products before smoking and after smoking and manufacturing. 
Table 5. Level of OCPs and PCBs (above the LOD and LOQ values) in different smoked pork meat products ( $\mu \mathrm{g} / \mathrm{kg}$ ).

\begin{tabular}{|c|c|c|c|c|c|c|c|}
\hline Sample & & $\alpha-\mathrm{HCH}$ & Lindane & РСВ 28 & РCB 52 & РCВ 153 & $\Sigma 6 \mathrm{PCB}$ \\
\hline \multirow[b]{2}{*}{ Pancetta before smoking } & $\mathrm{X} \pm \mathrm{SD}$ & $5.1 \pm 1.1$ & $15.1 \pm 1.08$ & $3.3 \pm 0.3$ & $7.003 \pm 4.42$ & $3.8 \pm 0.1$ & \\
\hline & Range frequency & $\begin{array}{c}<\text { loq-6.2 } \\
33.3 \% \\
\end{array}$ & $\begin{array}{c}12.03-28 \\
100 \% \\
\end{array}$ & $\begin{array}{c}<\text { loq }-10.1 \\
50 \%\end{array}$ & $\begin{array}{c}<\text { loq }-10.005 \\
\quad 33.3 \%\end{array}$ & $\begin{array}{c}<\text { loq }-4.62 \\
33.3 \%\end{array}$ & $<$ loq-20.1 \\
\hline \multirow[b]{2}{*}{ Pancetta after traditional smoking } & $\mathrm{X} \pm \mathrm{SD}$ & \multirow{2}{*}{$<\operatorname{loq}$} & $12.5 \pm 6.6$ & \multirow{2}{*}{$<\operatorname{loq}$} & $7 \pm 6.0005$ & \multirow{2}{*}{$<$ loq } & \\
\hline & Range frequency & & $\begin{array}{c}2.2-26.3 \\
100 \%\end{array}$ & & $\begin{array}{c}<\text { loq-20 } \\
58.3 \%\end{array}$ & & $<$ loq-20 \\
\hline \multirow[b]{2}{*}{ Pancetta after industrial smoking } & $\mathrm{X} \pm \mathrm{SD}$ & \multirow{2}{*}{$<\operatorname{loq}$} & $12.11 \pm 1.08$ & \multirow{2}{*}{$<\operatorname{loq}$} & $4.003 \pm 2.02$ & 4 & \\
\hline & Range frequency & & $\begin{array}{c}8.003-14.19 \\
100 \%\end{array}$ & & $\begin{array}{l}<\text { loq-5 } \\
66.7 \%\end{array}$ & $\begin{array}{l}<\text { loq-4 } \\
16.7 \%\end{array}$ & $<$ loq-8 \\
\hline \multirow[b]{2}{*}{ Pancetta ripening after traditional smoking } & $\mathrm{X} \pm \mathrm{SD}$ & \multirow{2}{*}{$<\operatorname{loq}$} & $15 \pm 14.07$ & \multirow{2}{*}{$<\operatorname{loq}$} & $3.004 \pm 4.002$ & $4 \pm 1$ & \\
\hline & Range frequency & & $\begin{array}{c}3-46 \\
100 \% \\
\end{array}$ & & $\begin{array}{c}<\text { loq-9.907 } \\
41.7 \%\end{array}$ & $\begin{array}{l}<\text { loq-8 } \\
83.3 \%\end{array}$ & $<\operatorname{loq}-15.8$ \\
\hline \multirow[b]{2}{*}{ Pancetta ripening after industrial smoking } & $\mathrm{X} \pm \mathrm{SD}$ & $7.015 \pm 5$ & $12.13 \pm 5.03$ & $1.2 \pm 0.4$ & $1.8 \pm 0.2$ & $8.003 \pm 6.001$ & \\
\hline & Range frequency & $\begin{array}{l}<\text { loq-12 } \\
33.3 \%\end{array}$ & $\begin{array}{c}4.11-22.17 \\
100 \%\end{array}$ & $\begin{array}{c}<\text { loq }-1.7 \\
83.3 \%\end{array}$ & $\begin{array}{c}<\text { loq- } 6.001 \\
83.33 \%\end{array}$ & $\begin{array}{c}<\text { loq-10.005 } \\
25 \%\end{array}$ & $<\operatorname{loq}-10.2$ \\
\hline \multirow[b]{2}{*}{ Tenderloin before smoking } & $\mathrm{X} \pm \mathrm{SD}$ & $30 \pm 1$ & $18.15 \pm 8.02$ & \multirow{2}{*}{$<\operatorname{loq}$} & $3.001 \pm 2.00015$ & $6.005 \pm 4.001$ & \\
\hline & Range frequency & $\begin{array}{l}<\text { loq-34 } \\
33.3 \%\end{array}$ & $\begin{array}{c}3.12-28.16 \\
100 \%\end{array}$ & & $\begin{array}{l}<\text { loq }-5 \\
66.7 \%\end{array}$ & $\begin{array}{c}<\text { loq-10.006 } \\
\quad 83.3 \%\end{array}$ & $<$ loq-13.5 \\
\hline \multirow[b]{2}{*}{ Tenderloin after traditional smoking } & $\mathrm{X} \pm \mathrm{SD}$ & $15 \pm 4$ & $15.11 \pm 2.08$ & \multirow{2}{*}{$<\operatorname{loq}$} & $1.003 \pm 0.5$ & $3 \pm 1$ & \\
\hline & Range frequency & $\begin{array}{l}10-20 \\
66.7 \% \\
\end{array}$ & $\begin{array}{c}12.01-18.23 \\
100 \%\end{array}$ & & $\begin{array}{c}<\operatorname{loq}-2.004 \\
66.7 \%\end{array}$ & $\begin{array}{c}2-6 \\
100 \% \\
\end{array}$ & $<\operatorname{loq}-6.2$ \\
\hline \multirow[b]{2}{*}{ Tenderloin after industrial smoking } & $\mathrm{X} \pm \mathrm{SD}$ & \multirow{2}{*}{$<\operatorname{loq}$} & $11.16 \pm 8.03$ & \multirow{2}{*}{$<\operatorname{loq}$} & \multirow{2}{*}{$<\operatorname{loq}$} & $5.003 \pm 6.001$ & \\
\hline & Range frequency & & $\begin{array}{c}3.1-19.19 \\
100 \%\end{array}$ & & & $\begin{array}{c}<\operatorname{loq}-10.004 \\
\quad 66.7 \%\end{array}$ & $<$ loq-10.004 \\
\hline \multirow[b]{2}{*}{ Tenderloin ripening after traditional smoking } & $\mathrm{X} \pm \mathrm{SD}$ & \multirow[t]{2}{*}{$<\operatorname{loq}$} & $22.11 \pm 7.12$ & \multirow[t]{2}{*}{$<$ loq } & $4.003 \pm 2.001$ & \multirow[t]{2}{*}{$<$ loq } & \\
\hline & Range frequency & & $\begin{array}{c}15.004-33.31 \\
100 \%\end{array}$ & & $\begin{array}{c}1.001-7.005 \\
100 \%\end{array}$ & & $<$ loq-7.005 \\
\hline \multirow[b]{2}{*}{ Tenderloin ripening after industrial smoking } & $\mathrm{X} \pm \mathrm{SD}$ & \multirow[t]{2}{*}{$<\operatorname{loq}$} & $11.17 \pm 8.03$ & $3 \pm 1$ & 20 & \multirow[t]{2}{*}{$<\operatorname{loq}$} & \\
\hline & Range frequency & & $\begin{array}{l}10-23 \\
100 \%\end{array}$ & $\begin{array}{c}2-4 \\
45.5 \%\end{array}$ & $\begin{array}{c}<\operatorname{loq}-20 \\
9.1 \%\end{array}$ & & $<\operatorname{loq}-20.4$ \\
\hline
\end{tabular}


Table 5. Cont.

\begin{tabular}{|c|c|c|c|c|c|c|c|}
\hline Sample & & $\alpha-\mathrm{HCH}$ & Lindane & РСB 28 & PCB 52 & РСB 153 & $\Sigma 6 \mathrm{PCB}$ \\
\hline \multirow[b]{2}{*}{ Dry neck before smoking } & $\mathrm{X} \pm \mathrm{SD}$ & $9 \pm 0.7$ & $17 \pm 3$ & \multirow{2}{*}{$<\operatorname{loq}$} & \multirow{2}{*}{$<\operatorname{loq}$} & $3 \pm 1$ & \\
\hline & Range frequency & $\begin{array}{c}<\text { loq }-20.02 \\
33.3 \%\end{array}$ & $\begin{array}{c}11.11-23.23 \\
100 \%\end{array}$ & & & $\begin{array}{c}<\operatorname{loq}-5.005 \\
75 \%\end{array}$ & $<\operatorname{loq}-5.005$ \\
\hline \multirow[b]{2}{*}{ Dry neck after traditional smoking } & $\mathrm{X} \pm \mathrm{SD}$ & \multirow{2}{*}{$<\operatorname{loq}$} & $11.15 \pm 1.14$ & \multirow{2}{*}{$<\operatorname{loq}$} & $3.003 \pm 1.004$ & 4.004 & \\
\hline & Range frequency & & $\begin{array}{c}4.003-31.46 \\
100 \%\end{array}$ & & $\begin{array}{c}<\text { loq }-5.0099 \\
75 \%\end{array}$ & $\begin{array}{c}<\operatorname{loq}-4.004 \\
8.33\end{array}$ & $<\operatorname{loq}-9.1$ \\
\hline \multirow[b]{2}{*}{ Dry neck after industrial smoking } & $\mathrm{X} \pm \mathrm{SD}$ & 15.05 & $13.15 \pm 3.066$ & \multirow{2}{*}{$<\operatorname{loq}$} & $1.1 \pm 0.2$ & $3.007 \pm 1.006$ & \\
\hline & Range frequency & $\begin{array}{c}<\text { loq-15.05 } \\
33.3 \%\end{array}$ & $\begin{array}{c}11.002-17.26 \\
100 \%\end{array}$ & & $\begin{array}{c}<\text { loq- } 1.2 \\
66.7 \%\end{array}$ & $\begin{array}{c}2.002-5.02 \\
100 \%\end{array}$ & $2.002-6.2$ \\
\hline \multirow[b]{2}{*}{ Dry neck ripening after traditional smoking } & $\mathrm{X} \pm \mathrm{SD}$ & 8.05 & $15.15 \pm 2.1$ & $1 \pm 0.4$ & $1.003 \pm 0.15$ & $5.007 \pm 1.004$ & \\
\hline & Range frequency & $\begin{array}{c}<\text { loq- } 8.05 \\
33.3 \%\end{array}$ & $\begin{array}{c}12.02-16.28 \\
100 \%\end{array}$ & $\begin{array}{l}<\text { loq-2 } \\
66.7 \%\end{array}$ & $\begin{array}{c}<\text { loq-1.05 } \\
66.7 \%\end{array}$ & $\begin{array}{c}4.002-6.01 \\
100 \%\end{array}$ & $4.002-8.01$ \\
\hline \multirow[b]{2}{*}{ Dry neck ripening after industrial smoking } & $\mathrm{X} \pm \mathrm{SD}$ & \multirow{2}{*}{$<\operatorname{loq}$} & $15.02 \pm 2.01$ & \multirow{2}{*}{$<\operatorname{loq}$} & $1.004 \pm 0.101$ & $4.1 \pm 0.004$ & \\
\hline & Range frequency & & $\begin{array}{c}12.008-16.04 \\
100 \%\end{array}$ & & $\begin{array}{c}<\operatorname{loq}-1.005 \\
66.7 \%\end{array}$ & $\begin{array}{c}<\text { loq- } 4.2 \\
16.7 \%\end{array}$ & $<\operatorname{loq}-5.2$ \\
\hline \multirow[b]{2}{*}{ Sausages before smoking } & $\mathrm{X} \pm \mathrm{SD}$ & $7.007 \pm 5.005$ & $18.12 \pm 4.05$ & \multirow{2}{*}{$<\operatorname{loq}$} & $1.18 \pm 0.2$ & $1.08 \pm 0.6$ & \\
\hline & Range frequency & $\begin{array}{c}<\text { loq-12.012 } \\
33.3 \%\end{array}$ & $\begin{array}{c}14.04-22.22 \\
100 \%\end{array}$ & & $\begin{array}{c}<\text { loq-2.06 } \\
83.3 \%\end{array}$ & $\begin{array}{c}<\text { loq }-2.01 \\
25 \%\end{array}$ & $<\operatorname{loq}-4.1$ \\
\hline \multirow[b]{2}{*}{ Sausages after traditional smoking } & $\mathrm{X} \pm \mathrm{SD}$ & \multirow{2}{*}{$<\operatorname{loq}$} & $16.16 \pm 3.03$ & \multirow{2}{*}{$<\operatorname{loq}$} & $3.003 \pm 1.001$ & $2.02 \pm 0.01$ & \\
\hline & Range frequency & & $\begin{array}{c}10.1-20.2 \\
100 \%\end{array}$ & & $\begin{array}{c}<\text { loq-4.004 } \\
40 \%\end{array}$ & $\begin{array}{c}1.01-3.03 \\
50 \%\end{array}$ & $<\operatorname{loq}-7.1$ \\
\hline \multirow[b]{2}{*}{ Sausages after industrial smoking } & $\mathrm{X} \pm \mathrm{SD}$ & \multirow{2}{*}{$<\operatorname{loq}$} & $15.25 \pm 6.06$ & \multirow{2}{*}{$<\operatorname{loq}$} & $5 \pm 2$ & $3 \pm 0.4$ & \\
\hline & Range frequency & & $\begin{array}{c}8.20-24.31 \\
100 \%\end{array}$ & & $\begin{array}{c}2-7 \\
100 \%\end{array}$ & $\begin{array}{c}<\text { loq-3.3 } \\
25 \%\end{array}$ & $2-6.8$ \\
\hline \multirow[b]{2}{*}{ Sausages ripening after traditional smoking } & $\mathrm{X} \pm \mathrm{SD}$ & \multirow[t]{2}{*}{$<\operatorname{loq}$} & $25.27 \pm 6.05$ & \multirow[t]{2}{*}{$<\operatorname{loq}$} & \multirow[t]{2}{*}{$<\operatorname{loq}$} & \multirow[t]{2}{*}{$<\operatorname{loq}$} & \multirow[t]{2}{*}{$<\operatorname{loq}$} \\
\hline & Range frequency & & $\begin{array}{c}20.21-31.31 \\
100 \%\end{array}$ & & & & \\
\hline \multirow[b]{2}{*}{ Sausages ripening after industrial smoking } & $\mathrm{X} \pm \mathrm{SD}$ & \multirow[t]{2}{*}{$<\operatorname{loq}$} & $27.15 \pm 5.06$ & \multirow[t]{2}{*}{$<\operatorname{loq}$} & \multirow[t]{2}{*}{$<\operatorname{loq}$} & \multirow[t]{2}{*}{$<\operatorname{loq}$} & \multirow[t]{2}{*}{$<\operatorname{loq}$} \\
\hline & Range frequency & & $\begin{array}{c}21.08-31.24 \\
100 \%\end{array}$ & & & & \\
\hline
\end{tabular}




\section{Discussion}

European Commission (EC) Regulation 178/2006 [26] and European Commission (EC) Regulation 149/2008 [27] prescribed MRLs for DDT and metabolites in various meat products are set at the maximum level of $100 \mu \mathrm{g} / \mathrm{kg}$. The measured value for DDD was several times below the limits specified in the EU.

The maximum levels for PCBs residues in the EU were set in 2006 [28], allowing maximum levels for sum of dioxins and dioxin-like PCBs expressed in World Health Organization (WHO) Toxic Equivalents (WHO-PCDD/F-PCB-TEQ) for pork and meat products at $1.5 \mathrm{pg} / \mathrm{g}$ fat. An amendment of European Commission (EC) 1125/2011 [29], with new limits based on WHO TEF (toxicity equivalency factors) from 2011 set the maximum permitted level of six PCBs at $40 \mathrm{ng} / \mathrm{g}$ fat for meat and pork products.

Food and Agriculture Organization (FAO) [30] set the maximum acceptable limits at $500 \mathrm{ng} / \mathrm{g}$ DDT and $300 \mathrm{ng} / \mathrm{g}$ for $\beta-\mathrm{HCH}$ and lindane. In addition, they determined the maximum total PCBs level at 200 ng/g lipid weight for PCBs congeners 28, 52, 101, 126, 138, 153 and 180. WHO [11] established TDI (tolerable daily intake) for total PCBs at $20 \mathrm{ng} / \mathrm{PCBs} / \mathrm{kg}$ of body weight per day.

The production and use of PCBs is either banned or restricted in most countries, but these compounds are still very important chemical pollutants found in the environment. They can easily migrate to the food chain, which makes them a significant hazard to human health. The presence of chemical contaminants, including OCPs and PCBs in pork muscle tissue and generally in animal tissues is a direct consequence of environmental pollution [23]. It can also be a result of contaminated pig feed [1]. In the past, the cases of PCBs occurrence in food of animal origin at concentrations higher than regulatory levels were connected with industrially produced animal feed. In recent years, the cases of PCBs level higher than the EU limit most commonly came from either "free range" animals, with the unknown source of contamination [31], or animal feed containing ingredients contaminated with PCBs [32,33]. A case where the source of contamination was determined was an old farm tank stained with PCBs day that peeled off at the place where the pigs were kept [34]. Adequate management can significantly reduce the exposure to PCBs.

The major sources of contamination of food of animal origin with PCBs are the following: PCBs from previously contaminated land, PCBs emitted from buildings and PCBs present on farms [31].

The OCPs and PCBs detected in the examined products at the end of the smoking process were $\alpha-\mathrm{HCH}$, lindane, PCB 28, 52 and 153 while other contaminants were below the detection limit (Table 5). In this study raw material pollutant content was low, but higher than the concentration of the examined contaminants in the products. This is most likely due to free range pig production and smoking process which can affect the concentration of OCPs and PCBs. According to the results obtained by Zabik et al. [35], smoking resulted in significantly higher reductions $(40-50 \%)$ in OCPs and PCBs levels than other thermal treatments used during culinary processing (baking, cooking). Also, higher concentrations of OCPs and PCBs detected in products after smoking than in the raw material can be explained by higher water content and lower fat content in the pre-smoked samples compared to the post-smoked samples. This was confirmed in the case of PAHs content [1], which at the end of the production process was higher than after the smoking process. Škaljac et al. [36], also found that the concentration of PAHs increases with the length of storage time in traditionally produced sausages.

Some studies have shown higher levels of PCBs than allowed. In the analyzed samples [37], five out of six PCBs indicators were above the recommended maximum limit of $15 \mu \mathrm{g} / \mathrm{kg}$ of fat.

Dioxin-like PCBs (DL-PCBs), including 6 indicator PCBs (PCBs 28, 52, 101, 138, 153, and 180) in meat products analyzed in Germany [38], WHO-PCB-TEQ ranged from $0.06 \mathrm{ng} / \mathrm{kg}$ fat for raw ham to $0.13 \mathrm{ng} / \mathrm{kg}$ fat for raw sausage. The most common were PCB 118, PCB 126, and PCB 156. In the study conducted in Albania [39], the PCBs profile was the following: PCB $153>$ PCB $52>$ PCB $138>$ PCB 180. The average PCBs content was $6 \mathrm{ng} / \mathrm{g}$. Costabeber et al. [40] examined the concentration of six PCBs in 55 samples of meat (pork and beef) and meat products (different types of sausages, salami, canned meat) from Brazil. They found the following concentrations: PCB $52(5.18 \mathrm{ng} / \mathrm{g}$ fat $)>$ PCB 180 $(1.69 \mathrm{ng} / \mathrm{g}$ fat $)>$ PCB 101 (1.35 ng/g fat) > PCB $28(1.19 \mathrm{ng} / \mathrm{g}$ fat $)>$ PCB153 (0.47 ng/g fat) > PCB 138 
$(0.43 \mathrm{ng} / \mathrm{g}$ of fat). The sum of 6 congeners was $10.3 \mathrm{ng} / \mathrm{g}$. Meat products have a higher PCBs content than meat (the highest content was in the products made from several types of meat, followed by pork, and the lowest was in cattle). The results of a study conducted by Boada et al. [41] showed that the consumption of meat and sausages increases the risk of DL-PCBs detection in human serum.

OCPs and PCBs belong to the environmental pollutants of anthropogenic origin [42]. Production and use of PCBs is practically completely stopped today, but their remains can still be found primary in electrical installations and the environment, and therefore in food. PCBs have been detected in fish from the Mediterranean 30 years after their ban [43]. DL-PCBs can form during cooking meat. Dong et al. [44] found that the concentrations of DL-PCBs in cooked beef was lower than those in raw beef, but a relatively high concentration of PCBs was detected in the oil vapors generated during meal preparation.

In addition to the concentrations measured in meat and its products, it is also necessary to calculate TDI. TDI for 6PCBs at $10 \mathrm{ng} / \mathrm{kg}$ bw $(0.01 \mu \mathrm{g} / \mathrm{kg} \mathrm{bw})$ [45-47]. The calculation for a $70 \mathrm{~kg}$ man is the following: daily intake of $\Sigma 6$ PCBs $(\mathrm{ng} / \mathrm{kgbw})=$ conc. $\Sigma 6 \mathrm{PCBs}(\mathrm{ng} / \mathrm{g}) \times \mathrm{x}(\mathrm{g}) / 70(\mathrm{~kg})$.

However, most OCPs have not been in use in the EU for many years, but they are still detected in food chain because of their persistence in the environment. Also, the risk can be unconscious and illegal use of OCPs which can lead to food poisoning.

In the present study, $\alpha-\mathrm{HCH}$ and lindane were the only OCPs detected under the LOQ values. Lindane $(\gamma-\mathrm{HCH})$ is the most toxic and the least stable of the $\mathrm{HCH}$ compounds. It is transformed into more stable $\alpha-\mathrm{HCH}$ over the time [48]. Similar results to these were obtained in a study conducted by Pine and Nuro [39]. They found that average pesticide concentration in meat samples is $13.5 \mathrm{ng} / \mathrm{g}$, while the lowest concentration was measured in pork samples. They also found that the presence of $\mathrm{HCHs}$ pesticides was a result previous use of lindane in agriculture.

The report on official controls conducted by Iceland and Norway in 2016 and the results obtained indicate that the potential of EU citizens being exposed to pesticide residues at a concentration that would have a negative impact on health is very low. Products of animal origin (pig fat), were analyzed for the presence of 22 pesticides. 11 of them were below the LOQ, while the remaining 11 were detected and qualified. The most commonly detected and quantified were DDT, hexachlorobenzene and chlordane. The MRL exceeded by a small amount in pig fat samples. For the pesticides for which MRLs are not prescribed, a default MRL of $0.01 \mathrm{mg} / \mathrm{kg}$ applies. 11 pesticides were sporadically detected, including DDT, hexachlorobenzene and chlordane in $2.8 \%, 1.8 \%$ and $1 \%$, while the others were present in $0.4 \%$ of samples (mainly pesticides whose use is not allowed due to their persistence: dieldrin, $\alpha-\mathrm{HCH}$, lindane). Endosulfane, heptachlor, $\beta-\mathrm{HCH}$ and methoxychlor were not detected in any samples. During 2016, 919 pig fat samples were analyzed, with $97.2 \%$ of samples having a pesticide concentration lower than the quantification limit. $2.8 \%$ of samples contained one or more pesticides. Up to three different residues were detected and quantified in four samples (0.4\%) [49].

As the methodology in analytical chemistry is evolving [50], the number of incidents related to food contamination is expected to increase.

Studies regarding the effect of culinary food processing on OCP concentration showed a significant decrease in OCP levels during thermal processing of food of animal origin [51,52]. Frying, roasting, grilling, cooking and other forms heat treatment of raw meat are effective in reducing pesticide levels in food [53,54]. Concentrations of OCPs and PCBs are associated with the fat content of food. During heat treatment of food, fat is reduced or released from the product, so there is less fat in the final product. Muresan et al. [52] noted that OCPs content decreased only by $1 \%$ during cold smoking. After the combined treatment of warm smoke and pasteurization, a reduction of $15 \%$ to $16 \%$ occurred. Roasting decreased OCPs by $46-56 \%$, respectively. In a review paper [55], it was also concluded that culinary procedures can modify the concentration of contaminants in food. However, this depends on the initial OCPs content in the treated food. Thermal treatments that release or remove fat can reduce the overall concentration of OCPs and PCBs in treated meat and products. 
In conclusion, concentrations of $\alpha-\mathrm{HCH}$, lindane, PCB 28, PCB 52 and PCB 153 were detected and quantified. All these values are under the prescribed maximum residual levels. Low levels of contaminants tested could be attributed to increased awareness of pesticides usage and their more proper use, i.e., compliance with the ban on its use, which results in reduction of their presence in the environment. The concentrations of OCPs and PCBs were not significantly affected by product type and by conditions of production. However, further study is required in order to monitor the presence and concentrations of OCPs and PCBs in these products.

Author Contributions: Conceptualization, K.M. and K.H.; Methodology K.M., B.K. and J.V.; Writing-original draft preparation, D.L.P. and N.N.; Data Curation, D.L.P. and L.P.; Writing-review and editing, D.L.P., N.N., and B.K.; Supervision, K.M. All authors have read and agreed to the published version of the manuscript.

Funding: This research was supported by the Ministry of Education, Science and Technological Development, Republic of Serbia; grant number TR 31011/2011-2019.

Conflicts of Interest: The authors declare no conflict of interest.

\section{References}

1. Mastanjević, K.; Kartalović, B.; Petrović, J.; Novakov, N.; Puljić, L.; Kovačević, D.; Jukić, M.; Lukinac, J.; Mastanjević, K. Polycyclic aromatic hydrocarbons in the traditional smoked sausage Slavonska kobasica. J. Food Compos. Anal. 2019, 83, 103282. [CrossRef]

2. Babić, J.M.; Kartalović, B.D.; Škaljac, S.; Vidaković, S.; Ljubojević, D.; Petrović, J.M.; Ćirković, M.A.; Teodorović, V. Reduction of polycyclic aromatic hydrocarbons in common carp meat smoked in traditional conditions. Food Addit. Contam. B 2018, 11, 208-213. [CrossRef] [PubMed]

3. Ljubojević, D.; Radosavljević, V.; Pelić, M.; Đorđević, V.; Živkov-Baloš, M.; Ćirković, M. Fatty acid composition, chemical composition and processing yield of traditional hot smoked common carp (Cyprinus carpio, L). Iran. J. Fish Sci. 2016, 15, 1293-1306.

4. Tiwo, C.T.; Tchoumbougnang, F.; Nganou, E.; Pankaj, K.; Nayak, B. Effect of different smoking processes on the nutritional and polycyclic aromatic hydrocarbons composition of smoked Clarias gariepinus and Cyprinus carpio. Food Sci. Nutr. 2019, 7, 2412-2418. [CrossRef] [PubMed]

5. Tompson, L.; Wageh, S.D. Environmental Chemical Contaminants in Food: Review of a Global Problem. J. Toxicol. 2019, 2019, 2345283. [CrossRef] [PubMed]

6. Fasano, E.; Yebra-Pimentel, I.; Martínez-Carballo, E.; Simal-Gándara, J. Profiling, distribution and levels of carcinogenic polycyclic aromatic hydrocarbons in traditional smoked plant and animal foods. Food Control 2016, 59, 581-590. [CrossRef]

7. Hitzel, A.; Pöhlmann, M.; Schwägele, F.; Speer, K.; Jira, W. Polycyclic aromatic hydrocarbons (PAH) and phenolic substances in meat products smoked with different types of wood and smoking spices. Food Chem. 2013, 139, 955-962. [CrossRef]

8. Santos, C.; Gomes, A.; Roseiro, L.C. Polycyclic aromatic hydrocarbons incidence in Portuguese traditional smoked meat products. Food Chem. Toxicol. 2011, 49, 2343-2347. [CrossRef]

9. Wretling, S.; Eriksson, A.; Eskhult, G.A.; Larsson, B. Polycyclic aromatic hydrocarbons (PAHs) in Swedish smoked meat and fish. J. Food Compos. Anal. 2010, 23, 264-272. [CrossRef]

10. Lauby-Secretan, B.; Loomis, D.; Grosse, Y. Carcinogenicity of polychlorinated biphenyls and polybrominated biphenyls. Lancet Oncol. 2013, 14, 287-288. [CrossRef]

11. World Health Organization. Polychlorinated Biphenyls: Human Health Aspects, Concise International Chemical Assessment Document 55. 2003. Available online: http://www.who.int/ipcs/publications/cicad/en/ cicad55.pdf (accessed on 17 November 2019).

12. Wang, H.S.; Sthiannopkao, S.; Du, J.; Chen, Z.J.; Kim, K.W.; Yasin, M.S.M.; Hashim, J.H.; Wong, C.K.; Wong, M.H. Daily intake and human risk assessment of organochlorine pesticides (OCPs) based on Cambodian market basket data. J. Hazard. Mater. 2011, 192, 1441-1449. [CrossRef] [PubMed]

13. Domingo, J.L. Concentrations of environmental organic contaminants in meat and meat products and human dietary exposure: A review. Food Chem. Toxicol. 2017, 107, 20-26. [CrossRef] [PubMed]

14. Carpenter, D.O. Polychlorinated biphenyls (PCBs): Routes of exposure and effects on human health. Rev. Environ. Health 2006, 21, 1-24. [CrossRef] [PubMed] 
15. Li, X.; Gan, Y.; Yang, X.; Zhou, J.; Dai, J.; Xu, M. Human health risk of organochlorine pesticides (OCPs) and polychlorinated biphenyls (PCBs) in edible fish from Huairou Reservoir and Gaobeidian Lake in Beijing, China. Food Chem. 2008, 109, 348-354. [CrossRef]

16. Muir, D.; Sverko, E. Analytical methods for PCBs and organochlorine pesticides in environmental monitoring and surveillance: A critical appraisal. Anal. Bioanal. Chem. 2006, 386, 769-789. [CrossRef]

17. Keller, J.M.; Swarthout, R.F.; Carlson, B.K.; Yordy, J.; Guichard, A.; Schantz, M.M.; Kucklick, J.R. Comparison of five extraction methods for measuring PCBs, PBDEs, organochlorine pesticides, and lipid content in serum. Anal. Bioanal. Chem. 2009, 393, 747-760. [CrossRef]

18. Björklund, E.; von Holst, C.; Anklam, E. Fast extraction, clean-up and detection methods for the rapid analysis and screening of seven indicator PCBs in food matrices. TrAC-Trend. Anal. Chem. 2002, 21, 40-53. [CrossRef]

19. Kartalović, B.; Mihaljev, Ž.; Prica, N.; Jakšić, S.; Ćirković, M.; Živkov Baloš, M. Method validation for determination of organochlorine pesticide residues in food and feed. Arch. Vet. Med. 2015, 8, 37-46.

20. European Commission. SANCO/12571/2013 of 19 November 2013. Guidance Document on Analytical Quality Control and Validation Procedures for Pesticide Residues Analysis in Food and Feed, Supersedes SANCO/12495/2011; Implemented by 1 January 2014; European Commission: Brussels, Belgium, 2013.

21. Anastassiades, M.; Lehotay, S. Fast and easy multiresidue method employing acetonitrile extraction/partitioning and "dispersive solid-phase extraction" for the determination of pesticide residues in produce. J. AOAC Int. 2003, 86, 412-431.

22. Kartalović, B.; Novakov, N.J.; Mihaljev, Ž.; Petrović, J.; Prica, N.; Babić, J.; Ćirković, M.A. Organochlorine pesticides in canned tuna and sardines on the Serbian market. Food Addit. Contam. B 2016, 9, 299-304. [CrossRef]

23. Pelić, M.; Kartalović, B.; Đorđević, V.; Puvača, N.; Teodorović, V.; Ćirković, M.; Ljubojević Pelić, D. Occurrence and dietary exposure of organochlorine pesticides in common carp obtained from integrated production systems. Food Addit. Contam. B 2019, 12, 303-309. [CrossRef] [PubMed]

24. Magnusson, B.; Örnemark, U. Eurachem Guide: The Fitness for Purpose of Analytical Methods-A Laboratory Guide to Method Validation and Related Topics, 2nd ed.; Eurachem: Torino, Italy, 2014; Available online: http://eurachem.org (accessed on 20 November 2019).

25. European Commission. SANTE/11813/2017. Guidance Document on Analytical Quality Control and Method Validation Procedures for Pesticide Residues and Analysis in Food and Feed. Implemented by 01/01/2018. Directorate General for Health and Food Safety. Available online: https://ec.europa.eu/food/sites/food/files/ plant/docs/pesticides_mrl_guidelines_wrkdoc_2017-11813.pdf (accessed on 20 November 2019).

26. European Commission. 178/2006. Commission regulation no 178/2006 EC of 1 February 2006 amending regulation no 396/2005 EC of the European parliament and of the council to establish annex I listing the food and feed products to which maximum levels for pesticide residues apply. Off. J. Eur. Union L. 2006, 29, 3-25.

27. European Commission. 149/2008. Commission regulation no 149/2008 EC of 29 January 2008 amending regulation no 396/2005 EC of the European parliament and of the council by establishing annexes II, III and IV setting maximum residue levels for products covered by annex I thereto. Off. J. Eur. Union L. 2008, 58, 1-398.

28. Commission Regulation. 1881/2006 of 19 December 2006 of 19 December 2006 setting maximum levels for certain contaminants in foodstuff. Off. J. Eur. Union L. 2006, 364, 5-24.

29. European Commission. Commission Regulation 1259/2011 of 2 December 2011 amending Regulation (EC) No 1881/2006 as regards maximum levels for dioxins, dioxin-like PCBs and non dioxin-like PCBs in foodstufs. Off. J. Eur. Union L. 2011, 62, 212-217.

30. Food and Agriculture Organization. Compilation of Legal Limits for Hazardous Substances in Fish and Fishery Products; FAO Fishery Circular No. 464; Food and Agriculture Organization: Roma, Italy, 1983; pp. 5-100.

31. Weber, R.; Herold, C.; Hollert, H.; Kamphues, J.; Blepp, M.; Ballschmiter, K. Reviewing the relevance of dioxin and PCB sources for food from animal origin and the need for their inventory, control and management. Environ. Sci. Eur. 2018, 30, 42. [CrossRef] [PubMed]

32. Heres, L.; Hoogenboom, R.; Herbes, R.; Traag, W.; Urlings, B. Tracing and analytical results of the dioxin contamination incident in 2008 originating from the Republic of Ireland. Food Addit. Contam. A Chem. Anal. Control Expo. Risk Assess. 2010, 27, 1733-1744. [CrossRef] [PubMed]

33. Marnane, I. Comprehensive environmental review following the pork PCB/dioxin contamination incident in Ireland. J. Environ. Monit. 2012, 14, 2551-2556. [CrossRef] [PubMed] 
34. Vaccher, V.; Marchand, P.; Picherot, M.; Dervilly-Pinel, G.; Lesquina, E.P.; Brosseaud, A.; Venisseau, A.; Le Bizeca, B. Field investigation to determine the environmental source of PCBs in a pig farm. Food Chem. 2018, 245, 394-400. [CrossRef]

35. Zabik, M.E.; Booren, A.; Zabik, M.J.; Welch, R.; Humphrey, H. Pesticide residues, PCBs and PAHs in baked, charbroiled, salt boiled and smoked Great Lakes lake trout. Food Chem. 1996, 55, 231-239. [CrossRef]

36. Škaljac, S.; Petrović, L.; Tasić, T.; Ikonić, P.; Jokanović, M.; Tomović, V.; Džinić, N.; Šojić, B.; Tjapkin, A.; Škrbić, B. Influence of smoking in traditional and industrial conditions on polycyclic aromatic hydrocarbons content in dry fermented sausages (Petrovská klobása) from Serbia. Food Control 2014, 40, 12-18. [CrossRef]

37. Weber, R.; Bell, L.; Watson, A.; Petrlik, J.; Paun, M.C.; Vijgen, J. Assessment of pops contaminated sites and the need for stringent soil standards for food safety for the protection of human health. Environ. Pollut. 2019, 249, 703-715. [CrossRef] [PubMed]

38. Andrée, S.; Jira, W.; Schwind, K.H.; Wagner, H.; Schwägele, F. Chemical safety of meat and meat products. Meat Sci. 2010, 86, 38-48. [CrossRef] [PubMed]

39. Pine, O.; Nuro, A. Organochlorine Pesticides and PCB in Meat and by-Products from Albanian Markets. J. Int. Environ. Appl. Sci. 2016, 11, 401-408.

40. Costabeber, I.; dos Santos, J.S.; Xavier, A.O.; Weber, J.; Leães, F.L.; Junior, S.B.; Emanuelli, T. Levels of polychlorinated biphenyls (PCBs) in meat and meat products from the state of Rio Grande do Sul, Brazil. Food Chem. Toxicol. 2006, 44, 1-7. [CrossRef]

41. Boada, L.D.; Sangil, M.; Álvarez-León, E.E.; Hernández-Rodríguez, G.; Henríquez-Hernández, L.A.; Camacho, M.; Zumbado, M.; Serra-Majem, L.; Luzardo, O.P. Consumption of foods of animal origin as determinant of contamination by organochlorine pesticides and polychlorobiphenyls: Results from a population-based study in Spain. Chemosphere 2014, 114, 121-128. [CrossRef]

42. Püssa, T. Toxicological issues associated with production and processing of meat. Meat Sci. 2013, 95, 844-853. [CrossRef]

43. Masci, M.; Nevigato, T. Non-dioxin-like PCBs: A survey on fishery and aquaculture from the Mediterranean area. Food Addit. Contam. B 2016, 9, 159-169. [CrossRef]

44. Dong, S.; Wu, J.; Liu, G.; Zhang, B.; Zheng, M. Unintentionally produced dioxin-like polychlorinated biphenyls during cooking. Food Control 2011, 22, 1797-1802. [CrossRef]

45. Opinion of the French Food Safety Agency. Establishment of Relevant Maximum Levels for Non Dioxin-Like Polychlorobiphenyls (NDL-PCB) in Some Food-Stuffs; AFSSA-Request No. 2006-SA-0305; AFSSA: Paris, France, 2007; pp. 1-27.

46. Vitenskapskomiteen for Mattrygghet. Opinion of the Panel on Contaminants of the Norwegian Scientific Committee for Food Safety; 07/505-3; Vitenskapskomiteen for Mattrygghet: Oslo, Norway, 2008.

47. Mihats, D.; Moche, W.; Prean, M.; Rauscher-Gabernig, E. Dietary exposure to non-dioxin-like PCBs of different popu-lation groups in Austria. Chemosphere 2015, 126, 53-59. [CrossRef]

48. Storelli, M.M.; Stuffler, R.G.; Marcotrigiano, G.O. Polycyclic aromatic hydrocarbons, polychlorinated biphenyls, chlorinated pesticides (DDTs), hexachlorocyclohexane, and hexachlorobenzene residues in smoked seafood. J. Food Prot. 2003, 66, 1095-1099. [CrossRef] [PubMed]

49. European Food Safety Authority. The 2016 European Union report on pesticide residues in food. EFSA J. 2018, 16, 5348.

50. Lopez-Garcia, M.; Romero-Gonzalez, R.; Frenich, A.G. Monitoring of organophosphate and pyrethroid metabolites in human urine samples by an automated method (TurboFlow ${ }^{\mathrm{TM}}$ ) coupled to ultra-high performance liquid chromatography-Orbitrap mass spectrometry. J. Pharm. Biomed. Alal. 2019, 173, 31-39. [CrossRef] [PubMed]

51. Sengupta, D.; Aktar, W.; Alam, S.; Chowdhury, A. Impact assessment and decontamination of pesticides from meat under different culinary processes. Environ. Monit. Assess. 2010, 169, 37-43. [CrossRef] [PubMed]

52. Muresan, C.; Covaci, A.; Socaci, S.; Suharoschi, R.; Tofana, M.; Muste, S.; Pop, A. Influence of Meat Processing on the Content of Organochlorine Pesticides. J. Food Process. Technol. 2015, 6, 11.

53. Domingo, J.L. Influence of Cooking Processes on the Concentrations of Toxic Metals and Various Organic Environmental Pollutants in Food: A Review of the Published Literature. Crit. Rev. Food Sci. Nutr. 2011, 51, 29-37. [CrossRef] 
54. Perelló, G.; Martí-Cid, R.; Llobet, J.M.; Castell, V.; Domingo, J.L. Concentrations of polybrominated diphenyl ethers, hexachlorobenzene and polycyclic aromatic hydrocarbons in various foodstuffs before and after cooking. Food Chem. Toxicol. 2009, 47, 709-715. [CrossRef]

55. Domingo, J.L.; Nadal, M. Carcinogenicity of consumption of red and processed meat: What about environmental contaminants? Environ. Res. 2016, 145, 109-115. [CrossRef]

(C) 2020 by the authors. Licensee MDPI, Basel, Switzerland. This article is an open access article distributed under the terms and conditions of the Creative Commons Attribution (CC BY) license (http://creativecommons.org/licenses/by/4.0/). 